\title{
Coupling power into accelerating mode of a three-dimensional silicon woodpile photonic band-gap waveguide
}

\author{
Ziran Wu, ${ }^{1,}{ }^{*}$ Robert Joel England, ${ }^{1}$ Cho-Kuen Ng, ${ }^{1}$ Benjamin Cowan, ${ }^{2}$ \\ Christopher McGuinness, ${ }^{3}$ Chunghun Lee, ${ }^{4}$ Minghao Qi ${ }^{4}$ and Sami Tantawi ${ }^{1}$ \\ ${ }^{1}$ SLAC National Accelerator Laboratory, 2575 Sand Hill Road, Menlo Park, California 95051, USA \\ ${ }^{2}$ Tech-X Corporation, 5621 Arapahoe Avenue, Boulder, Colorado 80303, USA \\ ${ }^{3}$ University of California San Francisco, 500 Parnassus Avenue, San Francisco, California 94143, USA \\ ${ }^{4}$ Purdue University, 101 North Grant Street, Purdue, Indiana 47907, USA
}

(Received 11 March 2014; published 19 August 2014)

\begin{abstract}
Silicon woodpile photonic crystals provide a base structure with which to build a three-dimensional dielectric waveguide system for high-gradient laser-driven acceleration. To realize an on-chip woodpile laser accelerator, a key component is the power coupler to deliver laser power to the fundamental accelerating mode. The woodpile waveguide is periodically loaded in the longitudinal direction; therefore simple cross-sectional mode profile matching is not sufficient to launch the accelerating mode appropriately and will result in significant scattering loss. Several traveling-wave coupler design schemes developed for multicell radio frequency cavity accelerators can be adapted to the woodpile accelerator coupler design. This paper presents design procedures and results using these methods. We present simulations indicating near 100\% power transmission between the transverse electric mode of a siliconguide side coupler and the transverse-magnetic-like accelerating mode of a woodpile waveguide. The coupler launches a full traveling-wave propagation of the accelerating mode, which maintains its propagation quality over long waveguide structures, and provides better tolerance on the structure fabrication uncertainty and material breakdown than standing-wave coupling.
\end{abstract}

\section{INTRODUCTION}

Utilizing the extraordinary electric fields and high repetition rates of modern solid state laser systems for charged particle acceleration has long been a goal of advanced accelerator research, due to the potential for higher accelerating gradient and reduced cost as compared with conventional rf accelerators [1,2]. Two critical requirements of direct laser acceleration are the presence of a longitudinal electric field along the particle beam trajectory and a speedof-light (SOL) phase velocity of the electric field. The latter is needed in order for the accelerating field to be synchronous with the relativistic particles. Several schemes for achieving this phase synchronous condition have been proposed that rely upon the inverse of various radiative processes, including the inverse Cerenkov accelerator $[3,4]$, the inverse free electron laser accelerator [5,6], and the inverse Smith-Purcell accelerator [7-9]. Another approach is to utilize dielectric near-field structures to confine a laser-induced accelerating mode within a narrow (width on the order of the laser wavelength) vacuum channel where the particles would

\footnotetext{
*wzr@slac.stanford.edu

Published by the American Physical Society under the terms of the Creative Commons Attribution 3.0 License. Further distribution of this work must maintain attribution to the author $(s)$ and the published article's title, journal citation, and DOI.
}

travel. These near-field approaches include the phase-reset grating accelerator $[1,10]$ and optical waveguide modes supported by dielectric photonic crystals $[2,11,12]$. We will refer to this latter method of particle acceleration as dielectric laser-driven acceleration (DLA). These structure-based laser accelerators have the advantages of near-field laser and particle interaction, which include wavelength scalability and better integration to create compact, multifunctional devices. A laser accelerator structure driven at an optical wavelength could potentially reduce the transverse footprint compared to current microwave-based accelerators by 4-5 orders of magnitude, and the longitudinal footprint by 2 or more orders of magnitude due to the higher acceleration gradients, implying significant cost reduction and feasibility of miniaturized devices. Dielectric laser accelerators employ dielectric materials rather than metals to build these structures, which is a proper choice due to the comparatively larger ohmic losses and lower breakdown thresholds of metal surfaces at optical wavelengths. Recently, near-infrared laser-induced relativistic and nonrelativistic electron acceleration has been experimentally demonstrated for the first time in a fused silica near-field DLA structure with maximum observed gradients of $300 \mathrm{MV} / \mathrm{m}$, an order of magnitude higher than typical operating gradients in conventional metallic structures $[9,13]$.

Dielectric photonic crystals are ideal media for building optical-scale waveguide systems for laser acceleration, because of their strong confinement of electric field in 
the waveguide [14], the higher damage threshold of dielectric material as compared to metal [15], and their compatibility with photolithographic fabrication methods to produce $\mu \mathrm{m}$-scale features $[10,16]$. SOL modes have been predicted in photonic crystal waveguides $[2,12]$ that furnish high gradient particle acceleration. Different than the grating DLA structure $[9,13]$, which is essentially a phase modulation mask to achieve net acceleration, these structures confine laser energy inside of a vacuum channel in an otherwise uniform but periodically varying dielectric material (or combination of materials) and propagate collinearly with the traversing charged particles, in true analogy to the metallic rf cavity accelerators. This energy confinement and copropagation ensure strong spatial overlap between the particles and the laser field, and therefore strong interaction.

Here we focus on a three-dimensional photonic crystal called the "woodpile" structure (WPS), made out of silicon and shown in Fig. 1. Silicon provides large index contrast to vacuum for light manipulation [14] and high breakdown threshold. Within its photonic band-gap frequency, the silicon woodpile structure provides full three-dimensional confinement of an electromagnetic wave. Therefore, necessary components for acceleration and particle/photon beam control, such as accelerating waveguide $[12,16]$, power coupler [17], focusing and deflecting structures [18-20], beam position monitors [20,21], and energy recycling resonators [22], can all be potentially built upon the same base lattice, and integrated on a single monolithic structure via lithographic fabrication [16,23]. We note that although this specific photonic crystal geometry is used in our calculations, our goal in the present paper is to develop a formalism and design methodology for efficient power transfer and coupling that can be more generally applied to a variety of three-dimensional photonic crystal waveguides and materials, and that can be scaled to multiple stages of acceleration.

The schematic in the inset in Fig. 1 shows a woodpile accelerating waveguide design first proposed in [12]. The base lattice consists of rectangular silicon rods stacked in a woodpile formation, whose collective scattering of light exhibits a three-dimensional photonic band gap. The structure possesses mirror symmetry about the $X Z$ plane, permitting a symmetric monopole mode for acceleration. This mode is confined to the open and roughly rectangular channel, as marked by the red break line in the Fig. 1 inset, and it propagates in the $z$ direction. The accelerator waveguide channel is $7 h$ tall and $3 a-w$ wide along its two transverse directions, with definition of dimensions $w$, $h$, and $a$ illustrated in the figure. The SOL synchronous mode is present with this waveguide channel design, which per calculation is able to provide $300 \mathrm{MV} / \mathrm{m}$ accelerating gradient with just $500 \mathrm{~W}$ of peak laser power at a laser wavelength of $1550 \mathrm{~nm}$ [24]. An integrated on-chip WPS accelerator is conceptually visualized in Fig. 1. An input

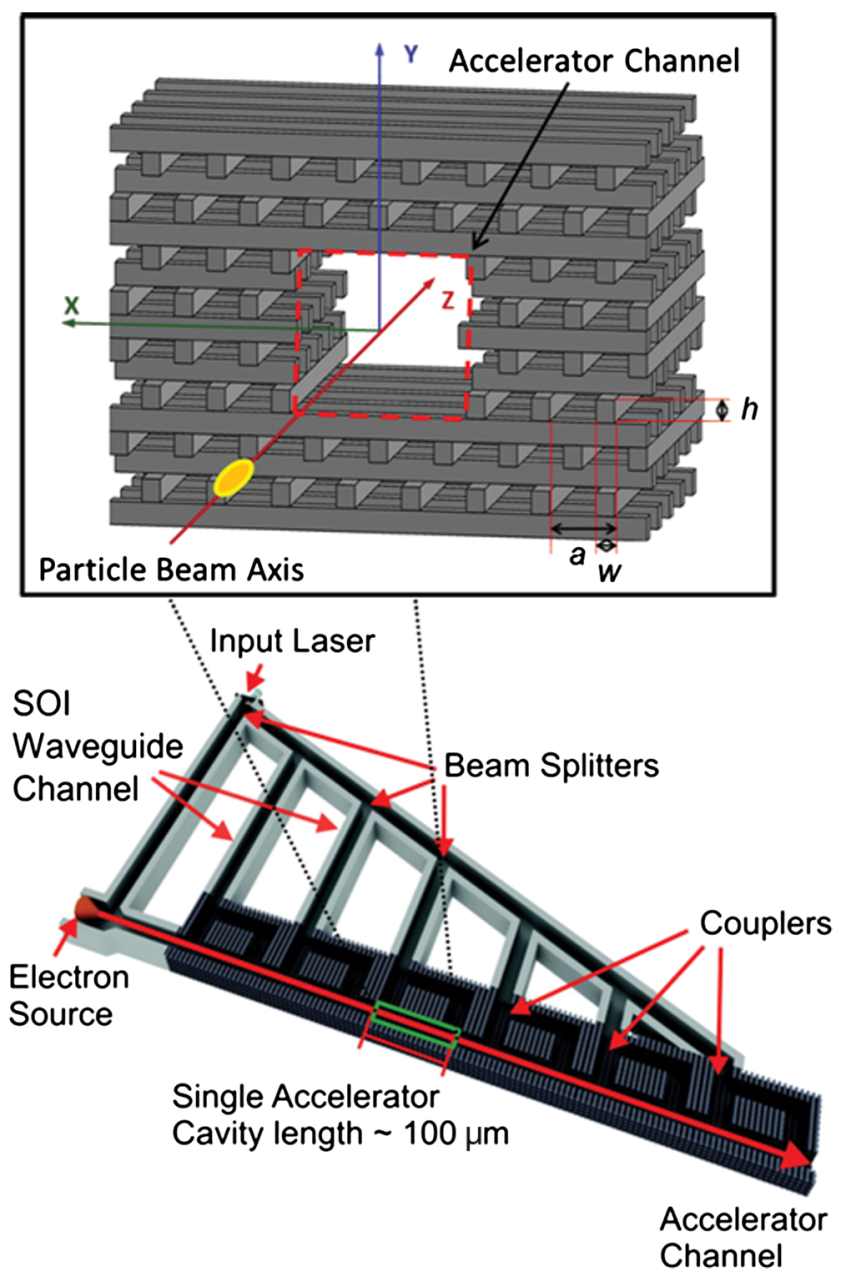

FIG. 1. Conceptual schematic of an on-chip woodpile accelerator consisting of multiple accelerator cavities. Inset: layout of a woodpile accelerator waveguide section supporting speed-of-light accelerating mode. Individual silicon rod's width, height, and spacing within each horizontal layer are denoted as $w, h$, and $a$, respectively.

laser pulse is split into different branches of silicon-oninsulator (SOI) waveguides, represented by solid black channels in the layout, to power multiple stages of woodpile accelerators (meshed cells in the layout). Electrons traversing through the accelerator channel get a kick at each cell by the laser field, and accelerate. The main channel for the input laser transport is tilted with respect to the accelerator channel to ensure the temporal overlap between electron bunch and laser pulse arrival times, due to the fact that the group velocity of the laser pulse in the waveguide is less than the speed of light $[12,19]$. Each accelerator stage contains a woodpile waveguide loop cavity in order to recycle the laser energy and enhance optical-to-beam efficiency [22], as the rectangular loops in the layout illustrate. Along the accelerator channel, sections of various functionalities, such as beam focusing and beam position monitor utilizing quadruple and dipolelike woodpile waveguide modes, could be built $[20,25]$. The SOI waveguides 
are basically silicon slabs sitting atop lower-refractiveindex materials, which confine laser light mostly within the silicon by total internal reflection. Splitters, bends, and combiners could be realized using SOI waveguides $[19,26]$ as the elements of this on-chip power distribution network.

From this conceptual layout, we can identify three distinct coupler designs that would constitute the basic subcomponents of the power distribution network: (1) couplings between the input laser and the SOI waveguide, (2) couplings between the SOI main line and side branches of laser transport, and (3) couplings between the SOI waveguide and the woodpile waveguide. Simulations relevant to components (1) and (2) have been presented elsewhere, showing coupling between the incident free-space or fiber laser and the SOI guide with $70 \%$ efficiency using nonperiodic silicon grating structure and a top-illumination coupling scheme, and 40\%-50\% demonstrated experimentally [19,27]. In addition, a 1 to $n$ splitter on a SOI network has also been realized [28]. Consequently, this work will focus on item (3), the coupler design to inject power from a SOI guide into the woodpile waveguide. Such a coupler needs to be compact, because coupling of a new laser pulse into the accelerating waveguide must occur about every 100 wavelengths. The reason for this requirement is that the group velocity of the accelerating mode is less than that of the speed of light, and therefore longitudinal overlapping of the laser pulse with the relativistic electron bunch is sustainable for a limited distance [24]. More importantly, the design shall ascertain that laser power is fully coupled to the desired accelerating mode while other unwanted woodpile waveguide modes are suppressed. This requirement becomes more challenging as the aperture size of the accelerator channel is enlarged to improve electron throughput, making the waveguide more overmoded. It is also tempting to design this coupler solely using silicon, so that its fabrication can be conveniently incorporated into the woodpile accelerator cell fabrication. One advantage of the woodpile photonic crystal lattice is that the orthogonally interlaced silicon rods discretize the structure geometry, and provide necessary degrees of freedom to perturb the electromagnetic fields in order to optimize coupling to the desired mode.

This paper is arranged as follows: Section II introduces the longitudinally periodic nature of a woodpile waveguide, and a periodic voltage-standing-wave-ratio (VSWR) method adapted from metallic rf cavity accelerators designed to evaluate the mode matching and internal scattering loss of a power coupler. Section III presents a traveling-wave launch method to circumvent standing waves in a longitudinally periodic waveguide, with one example of its successful application to a woodpile TE-like propagating mode. With these two methods established, Sec. IV combines them to make transverse electric (TE) to transverse magnetic (TM) side coupler designs for woodpile accelerating waveguides. The accelerating waveguide proposed in Ref. [12] is found to be overmoded after excitation by the side coupler. We introduce a new accelerating mode with a modified woodpile waveguide channel design to guarantee single-mode operation, and a side coupler design is demonstrated to launch this propagating mode without standing wave and with almost $100 \%$ power efficiency. Discussion and conclusion of this work follow. In the Appendix, fabrication of the woodpile accelerating waveguide using a new silicon-membrane-based method is proposed, with manufactured structures shown.

\section{LONGITUDINALLY PERIODIC WAVEGUIDE AND VSWR METHOD}

\section{A. Periodic VSWR formalism}

Design of power couplers for the woodpile accelerator requires understanding of the mode propagation in both the accelerating channel and side-coupled waveguide power feed. Unlike the two-dimensional case such as the SOI guide and the optical fiber, the woodpile waveguide is periodically loaded in the longitudinal direction, in close analogy to multicell cavity disk loaded rf accelerators. Because of this periodic longitudinal variation, a crosssectional matched incident mode profile will not be sufficient to launch the propagating mode in the woodpile guide appropriately, resulting in significant scattering loss. In fact, the incident mode profile of a specific phase only provides one of the space harmonics that compose the propagating mode in the woodpile waveguide, while all other harmonic phases are missing.

A periodic voltage-standing-wave-ratio method previously developed for disk-loaded rf waveguides [29] can be applied to woodpile waveguides to evaluate the internal scattering loss. The geometric periodicity in the longitudinal $z$ direction demands that the steady-state electric field in the woodpile waveguide also shows the same periodicity, i.e. between adjacent periods the electric fields have identical magnitude and are different only by a phase advance:

$$
E(z \pm P)=E(z), \quad \phi(z \pm P)=\phi(z) \pm \psi,
$$

where $P$ is the periodicity in the $z$ direction ( $a$ in the woodpile waveguide case) and $\psi$ is the phase advance per period. $E(\mathrm{z})$ and $\phi(z)$ are the magnitude and phase of the electric field, respectively, depending on the polarization direction of the mode in the waveguide.

Introducing the normalized backward wave amplitude $R$, the main component of the complex electric field vector in the woodpile waveguide consists of contributions from the forward and backward waves:

$$
E_{c}(z)=E(z)\{\exp [-j \phi(z)]+R \exp [j \phi(z)]\} .
$$

Defining the quantities

$$
\begin{aligned}
F^{ \pm}(z) & =E_{c}(z \pm P) / E_{c}(z), \\
\Sigma(z) & =F^{+}(z)+F^{-}(z), \quad \text { and } \\
\Delta(z) & =F^{+}(z)-F^{-}(z),
\end{aligned}
$$


some derivation will yield the following:

$$
\begin{aligned}
2 \cos (\psi) & =\Sigma(z), \\
R \exp [2 j \phi(z)] & =[2 \sin (\psi)-j \Delta(z)] /[2 \sin (\psi)+j \Delta(z)] .
\end{aligned}
$$

Equation (4) implies that $\Sigma(z)$, although defined by the sum of complex quantities in Eq. (3), is actually a real number and is independent of the $z$ coordinate, because the phase advance per period $\psi$ is real and independent of $z$ for a propagating mode. Also, the magnitude $|R|$ can be calculated from $\psi$ and the field quantity $\Delta(z)$ :

$$
|R|=\left|\frac{2 \sin (\psi)-j \Delta(z)}{2 \sin (\psi)+j \Delta(z)}\right| .
$$

As the normalized magnitude of the backward wave with respect to the forward wave magnitude, it is an important parameter to evaluate the scattering loss in the woodpile waveguide. Full traveling-wave propagation is reached when $|R|$ equals to zero, as opposed to a partial standingwave launch when $|R|$ is not zero and thus a backward wave exists.

\section{B. Benchmarking of the periodic VSWR method on a woodpile waveguide}

In order to show that the above equations are applicable to a WPS waveguide, a single-moded, linearly polarized silicon woodpile waveguide is modeled using Ansoft HFSS [30]. The structure, as depicted in Fig. 2(a), is one-quarter of the woodpile waveguide utilizing its 4-fold symmetries, excited by a back-to-back setup with two silicon guides inserted one period $a$ into it at the front and back apertures. The symmetry boundaries assigned to the model are a perfect electric (PEC) boundary at the front $Y Z$ plane and a perfect magnetic boundary at the bottom $X Z$ plane, so that the allowed modes in the simulation are TE-like modes polarized in the $x$ direction and propagating along the $z$ direction, in both the silicon guide and the woodpile waveguide. The woodpile structure is made of silicon with a dielectric constant of 11.9, and it is assumed that ohmic losses are negligible. The geometric parameters are $a=565 \mathrm{~nm}, w=158 \mathrm{~nm}$, and $h=200 \mathrm{~nm}$, matching the dimensions first introduced in Ref. [12]. The simulating wavelength of $1.55 \mu \mathrm{m}$ falls roughly in the middle of the structure's photonic band gap. The woodpile waveguide channel has a full aperture size of $a-w$ wide and $5 h$ tall, and the silicon guides are $a-w-40 \mathrm{~nm}$ wide and $h$ tall so they do not touch the woodpile silicon rods. The orange stubs at the end of each silicon guide are quarter-wave impedance matching layers (material of dielectric constant 3.45 and $210 \mathrm{~nm}$ thickness) to minimize reflection at the silicon/air interfaces. Electric and magnetic field plots confirm that the correct TE mode obtained from eigenmode simulations [30] is excited, and it is the only mode present
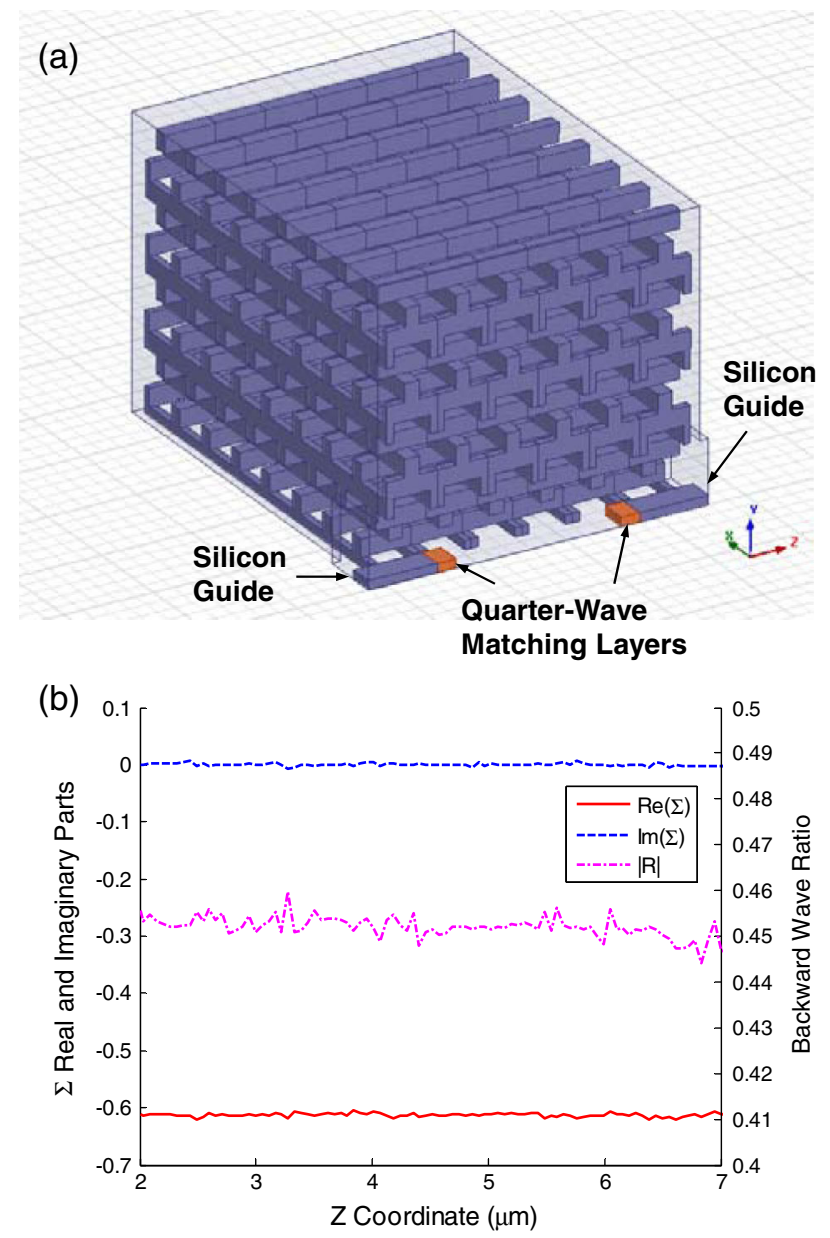

FIG. 2. (a) A TE-mode silicon woodpile waveguide excited by back-to-back silicon guide couplers. (b) Calculated $\Sigma(z)$ and the normalized backward wave amplitude $|R|$ in this woodpile waveguide.

in the woodpile waveguide under the applied boundary conditions and excitation.

The transverse electric field component $E_{x}(z)$ is then extracted along the center $(0,0, z)$ line of the woodpile waveguide channel, and used in Eq. (3) as the $E_{c}(z)$ quantity. The calculated $\Sigma(z)$ and normalized backward wave amplitude $|R|$ according to Eqs. (3) and (5) are then plotted in Fig. 2(b), with the abscissa being the longitudinal coordinate of the simulated 9 unit cells of the woodpile waveguide. From the plot, the real and imaginary parts of $\Sigma(z)$ (left ordinate) are indeed both flat lines along the waveguide longitudinal direction and are therefore independent of the $z$ coordinate. The imaginary part of $\Sigma(z)$ is very close to zero, confirming that it is a real number within the numerical uncertainty of the simulation. From the value of the real part of $\Sigma(z)$, the phase advance per period in this woodpile waveguide is $128^{\circ}$, agreeing with the value from the eigenmode simulation of this TE mode. From the value of $|R|$ (right ordinate), a standing wave is excited in the woodpile waveguide, with a backward propagating wave of 
about $45 \%$ of the magnitude of the forward propagating wave. This example validates application of the above formalism to silicon woodpile waveguides and use of the calculated $\psi$ and $|R|$ as figures of merit to evaluate the mode being excited and the quality of a traveling-wave launch.

\section{TRAVELING-WAVE LAUNCH METHOD}

\section{A. Mode launcher for traveling wave excitation}

A traveling-wave excitation of the fundamental accelerating mode in the woodpile waveguide is preferred to a standing-wave excitation for several reasons. The number of periods (woodpile waveguide cells) along the longitudinal direction would not alter the field propagation once a traveling wave is established. Therefore the structure design could be conveniently expanded to long waveguide lengths by simply adding woodpile cells, without having to simulate all the cells, which can quickly become challenging with limited computation resources, especially in threedimensional cases. A traveling-wave match usually has more bandwidth around the working frequency than a standing-wave match, since the coupling does not rely on resonances. For the same reason, the woodpile structure fabrication error is more tolerated in a traveling-wave match case [23]. A traveling-wave match may also reduce the likelihood of material breakdown under high laser power, because unlike the standing wave case, electromagnetic field maxima are not localized at fixed positions. A traveling-wave launch method based on scattering matrix theory has previously been developed for rf cavity accelerator structures [31], and we have adapted this approach to the woodpile waveguide.

The idea of mode launchers in a symmetric layout connecting a rf cavity waveguide of multiple cells is illustrated in Fig. 3(a). The mode launchers are waveguides of uniform cross section, such as conventional metallic

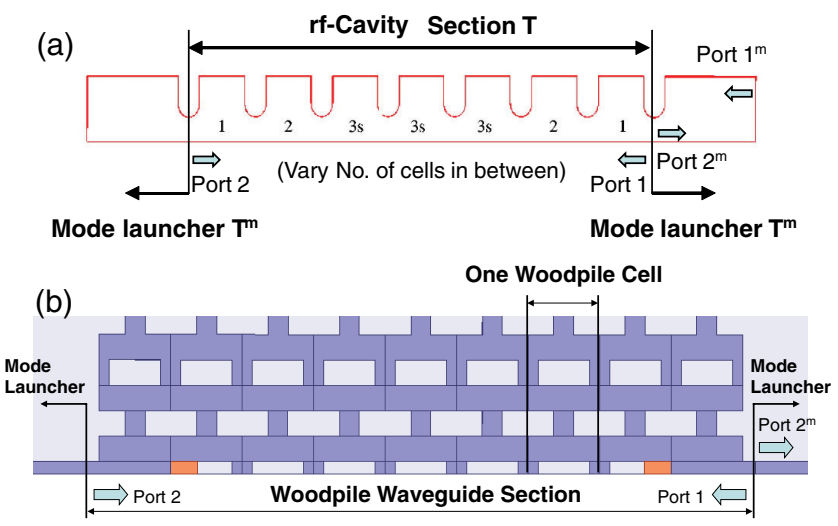

FIG. 3. (a) Mode launchers at the front and back of a multicell rf cavity waveguide to achieve traveling-wave match. (b) An equivalent configuration for a woodpile waveguide and back-toback silicon guide mode launchers. rectangular waveguides, or SOI guides in this on-chip woodpile accelerator case, with a transmission matrix $T^{m}$. They are back to back connected to a middle section in order to excite it. The middle section consists of a longitudinally periodic waveguide with $N$ periods and transmission matrix $T$. The total transmission matrix of the whole structure is given by the cascade product of its individual sections [31]:

$$
T^{\mathrm{tot}}=T^{m} T T^{m}, \quad S_{11}^{\mathrm{tot}}=T_{12}^{\mathrm{tot}} / T_{22}^{\mathrm{tot}}=0 .
$$

The second equation in Eq. (6) comes naturally from the requirement of a fully matched coupler. The matrix $S^{\text {tot }}$ in this notation refers to the corresponding scattering matrix of the transmission matrix $T^{\text {tot }}$. Defining $\left|S^{\mathrm{m}}\right|$ and $\phi^{\mathrm{m}}$ as magnitude and phase of the scattering matrix $S^{\mathrm{m}}$ of the mode launcher, it can be derived that [31]:

$$
\left|S_{22}^{\mathrm{m}}\right|=\frac{\cos \left(\phi_{11}+\phi_{22}^{\mathrm{m}}\right)}{\left|S_{11}\right|}-\sqrt{\left[\frac{\cos \left(\phi_{11}+\phi_{22}^{\mathrm{m}}\right)}{\left|S_{11}\right|}\right]^{2}-1}
$$

The magnitude and phase of the reflection coefficient $S_{11}$ can be obtained from a separate simulation of the periodic waveguide section in the middle. For a given complex $S_{11}$, Eq. (7) gives the explicit form of the magnitude $\left|S_{22}{ }^{\mathrm{m}}\right|$ as a function of its phase $\phi_{22}^{\mathrm{m}}$. Here $S_{22} \mathrm{~m}$ is the reflection coefficient looking into the mode launcher away from the middle rf cavity section. The next step is to add one or more periods to the middle section, simulate it, and thus a new $S_{11}$ is obtained. Then a second curve of $S_{22}{ }^{\mathrm{m}}$ magnitude vs phase can be drawn. In a traveling-wave match case, the propagating mode with all the space harmonics will be fully established regardless of the number of periods along the waveguide. Hence these two curves must intersect, giving one common point that tells the required $S_{22}{ }^{\mathrm{m}}$ to launch this traveling wave. The final step is to design the mode launcher by introducing a discontinuity into the two-dimensional input waveguide that can be adjusted to match the desired reflection coefficient $S_{22}{ }^{\mathrm{m}}$ at the intersection. One example can be found in [32] where three cases of different numbers of periods in the middle section are simulated, and the resultant three curves have a common intersection point specifying the required $S_{22}$ m to launch a traveling wave in the simulated disk-loaded rf cavity waveguide.

An analogy of this layout to the woodpile waveguide is illustrated in Fig. 3(b). The middle section now consists of multiple woodpile waveguide cells, equivalent to the rf cavity example in part (a) in the sense that they are both periodically loaded waveguides. The front and back inserted silicon guides provide the necessary twodimensional port mode excitation to feed the woodpile waveguide, from which the two-port $S$ parameters can be obtained via an HFSS waveport simulation [30]. The mode launchers attached to the inserted silicon guides are also 
silicon guides of identical transverse cross sections but are specially designed to provide the required $S_{22}{ }^{\mathrm{m}}$ to launch a traveling wave in the woodpile waveguide section.

\section{B. Traveling-wave match to a TE-mode woodpile waveguide}

Applying this method to the silicon woodpile waveguide, the simulation introduced in the layout of Fig. 2(a) is repeated, with the number of woodpile waveguide cells in the middle varied. The dimensions of the woodpile waveguide aperture and the silicon guide along the $x$ direction are also enlarged to $a+w-100 \mathrm{~nm}$ and $a+w-140 \mathrm{~nm}$, respectively, to allow more laser energy delivered under the same laser fluence. The simulation uses waveport excitation, with port modes still being the fundamental TE mode of the silicon guides, to obtain the $S$ parameters of the structure. With the simulated $S_{11}$ of the 2-, 3-, and 4-cell cases, three curves of the $S_{22}{ }^{\mathrm{m}}$ magnitude vs phase are drawn in Fig. 4(a). They indeed intersect at a common point, giving the desired $S_{22}$ m for a traveling-wave mode launcher to have 0.577 magnitude and $39.4^{\circ}$ phase.

In order to achieve this $S_{22}{ }^{\mathrm{m}}$, the two-dimensional silicon guides connecting the ends of the simulated woodpile waveguide structure are perturbed. As shown by the layout in Fig. 4(b), this perturbation is realized by embedding a chunk of perfect conductor in the silicon guide (red piece in the inset). We note that although a perfect conductor is chosen here to simplify the simulation, in a physical structure this would not be ideal due to the low breakdown thresholds of most high-conductivity metals. The required phase match perturbation on the input guide could be accomplished through a variety of alternative means, which are discussed in Sec. IVA. The depth of the chunk along the $x$ direction (denoted as $l_{x}$ ) provides direct control to tune the magnitude of $S_{22}{ }^{\mathrm{m}}$, whereas the distance between the PEC chunk and the front end of the silicon guide (denoted as $z_{0}$ ) determines its phase independently. Its thickness $l_{z}$ along the $z$ direction is set to $100 \mathrm{~nm}$. Separate port simulations are run on this perturbed silicon guide segment to determine these two dimensions, and $l_{x}=57.5 \mathrm{~nm}$ and $z_{0}=927 \mathrm{~nm}$ yield the desired $S_{22}{ }^{\mathrm{m}}$. After this mode launcher is designed, it is installed on both ends of the 4-cell woodpile waveguide middle section, as the main layout demonstrates, and the whole structure is simulated again. For this optimized configuration, we find that the total return loss $S_{11}{ }^{\text {tot }}$ is below $-20 \mathrm{~dB}$ and the total transmission $S_{12}{ }^{\text {tot }}$ is reaching $98.1 \%$.

To show that the mode launcher truly excites a traveling wave, the normalized backward wave amplitude $|R|$ is calculated using the on-axis $(0,0, z) E_{x}$ field extracted from the port simulation. The calculated $|R|$ in this example is less than 0.05 over the length of the waveguide. Therefore, an over $95 \%$ traveling wave match is achieved. For another case of seven woodpile waveguide cells as the middle section with the same mode launchers, the transmission is
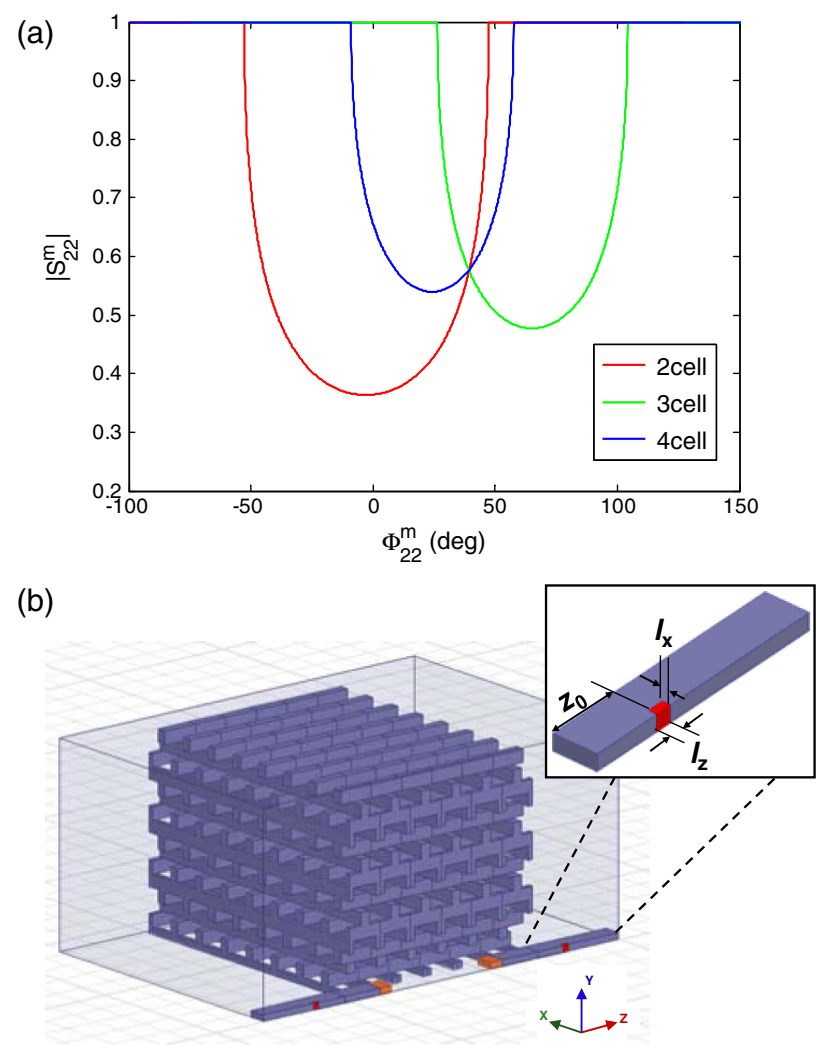

FIG. 4. (a) Multiple-cell simulation to determine the required $S_{22}{ }^{\mathrm{m}}$ for a traveling-wave match to a TE-mode woodpile waveguide. (b) The traveling-wave mode launcher design: perturbation in a silicon guide by a piece of perfect conductor, with its dimensions marked in the inset.

94.98\% with less than 5\% normalized backward wave amplitude as well. Therefore, there is truly a traveling wave launched in the woodpile waveguide and the coupling is nearly $100 \%$ regardless of the number of waveguide cells.

\section{POWER COUPLER FOR THE ACCELERATING MODES}

Now that the traveling-wave launch method is established for a TE-mode silicon woodpile waveguide, the next step is to apply it to a woodpile accelerating waveguide designed to support a TM-mode. Side coupling as shown in the on-chip accelerator layout in Fig. 3 introduces minimal obstacles along the electron trajectory, and is therefore preferred. If one of the original silicon rods of the base woodpile structure is enlarged in width (as noted $w$ in the Fig. 1 inset), it forms a nonuniform channel in the woodpile structure, and could serve as a silicon guide to send in laser power similar to the excitation silicon guides in Fig. 2(a). This widened silicon guide together with its adjacent woodpile structure form a "solid-core" woodpile waveguide in the band gap range, as compared to the "hollowcore" woodpile accelerator waveguide introduced in Sec. I. When it is linked perpendicularly to a woodpile 
accelerating waveguide to deliver power, ideally traveling waves need to be established both in the solid core and the hollow core, which are both periodically loaded in their respective longitudinal directions. Through simulations we have found that the aforementioned traveling-wave launch method is not applicable in the case that two hollow-core woodpile waveguides are coupled perpendicularly. Fortunately, the simulations also show that a confined TE-like mode is supported in the widened silicon guide but is slightly modified by the adjacent woodpile structure. This solid-core woodpile waveguide is then a quasi-2D waveguide, still pertinent for launching traveling waves in the woodpile accelerating waveguide section using the above scheme.

\section{A. Coupler design for the accelerating waveguide mode}

The layout of Fig. 5(a) illustrates a back-to-back power coupler design for a woodpile accelerating waveguide. The accelerating waveguide lies along the $x$ direction, and its dimensions follow the nominal design presented in Sec. I and Ref. [12]. The model is again a quartersymmetric structure, with its front $Y Z$ plane and bottom $Z X$ plane assigned the perfect magnetic boundary to
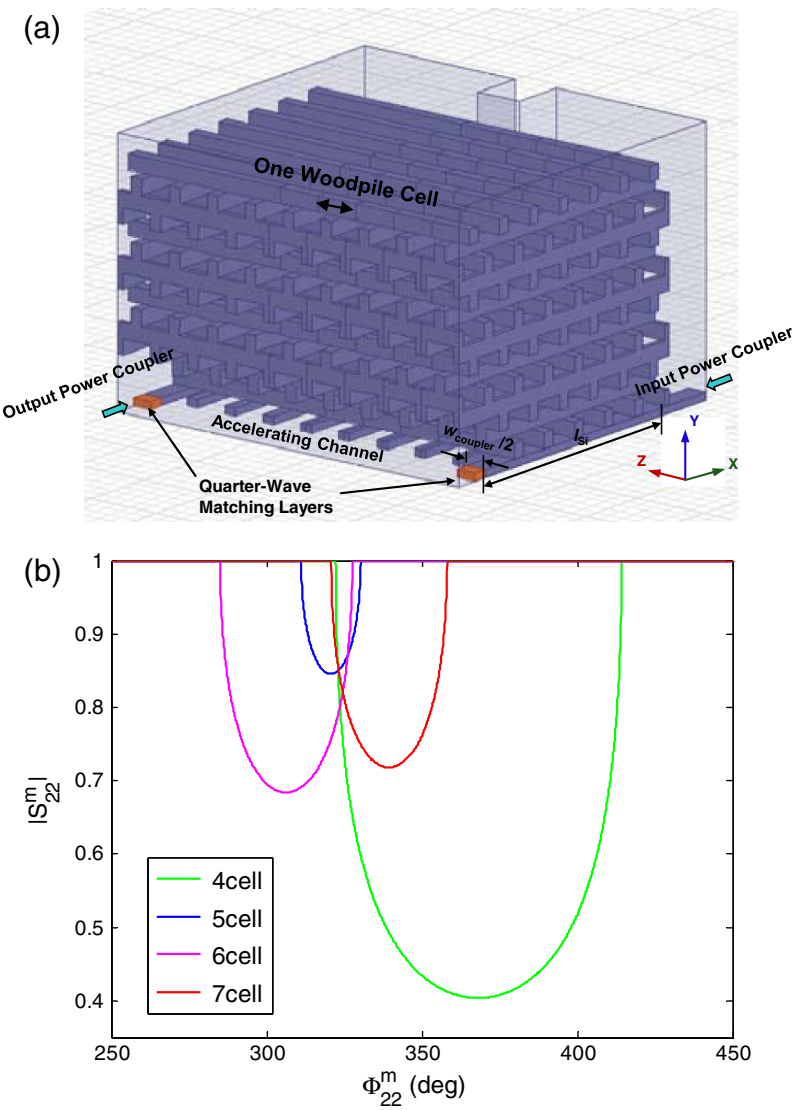

FIG. 5. (a) TE-to-TM mode coupler layout for a 7-cell woodpile accelerating waveguide, and (b) its multiple-cell simulation results to determine the required $S_{22}$ m for traveling-wave launch. simulate TM-like accelerating modes. A pair of these widened silicon rods, having a full width of $3.32 w$ but still $h$ tall, act as input and output side couplers at the beginning and end of the accelerating waveguide. Because of the perfect electric boundary condition assigned at the $X Y$ planes on the outer boundary of the model, a TE mode polarized in the $z$ direction is excited by a waveport in this coupler rod. This mode is then delivered into the accelerating waveguide, where TE mode to TM mode conversion happens at the coupling corner. The orange stubs shown in Fig. 5(a) at the end of each coupler rod are again quarter-wave impedance matching layers to minimize reflection at the silicon and air interfaces. We conduct these simulations at a design frequency of $197 \mathrm{THz}$, corresponding to a laser wavelength of $1.5 \mu \mathrm{m}$. This is a sensible choice for this example, since silicon is transmissive in this spectral region and mode-locked solid state lasers are available. This back-to-back setup forms the middle section that was shown in Fig. 3(b). By simulating the input port reflectance $\left(S_{11}\right)$ of this structure the $S_{22} \mathrm{~m}$ magnitude vs phase plot can be drawn again using Eq. (7), the result of which is shown in Fig. 5(b). The resultant curves of four different numbers of woodpile waveguide cells do not exactly have a common intersection point, however, indicating that a $100 \%$ traveling wave could not be launched. Our simulations indicate that the ending $x$ location of the coupler rods in the accelerating waveguide (denoted as $l_{\mathrm{Si}}$ ), as well as their rod width (denoted as $\left.w_{\text {coupler }}\right)$, are two direct parameters that tune the coupling. However, an extensive trial of different $l_{\mathrm{Si}}$ and $w_{\text {coupler }}$ values still could not locate a case where curves of different numbers of waveguide cells share a common point for traveling-wave propagation.

The failure of the traveling-wave launch scheme in this example is due to the overmoded condition in the woodpile accelerating waveguide. Through HFSS eigenmode simulations, we find that the accelerating waveguide geometry simulated in Fig. 5(a) actually supports two TM-like modes. Both of them are energy-confined propagating modes, and their dispersion curves both intersect with the speed-of-light line. Plotted in Fig. 6 are the electric field vector plot and the complex magnitude color map of their $E_{z}$ components, at the middle cut plane of one woodpile waveguide cell [as denoted in Figs. 3(b) and Fig. 5(a)] and with the same phase. In Fig. 6(a) is the fundamental accelerating mode with a relatively uniform and in-phase longitudinal $E_{z}$ component over the hollow core area, favoring the purpose of electron acceleration. Shown in Fig. 6(b) is the second spurious waveguide mode. This mode also possesses a significant longitudinal (z) component, but the field is nonuniformly distributed between two localized regions at the top and side of the hollow core (as displayed in the quarter-section view of Fig. 6), leaving only a small longitudinal $E_{z}$ component near the central area of the waveguide. This mode is inauspicious due to its 
(a)

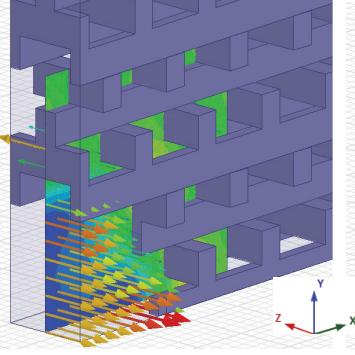

(b)

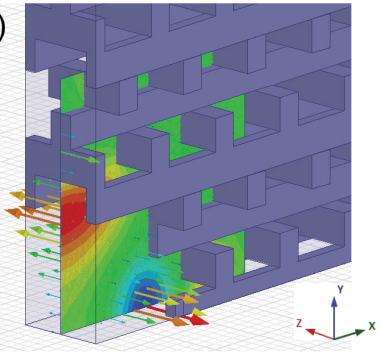

FIG. 6. Two TM-like speed-of-light modes of the original woodpile accelerating waveguide design: (a) the in-phase fundamental accelerating mode, (b) the out-of-phase spurious mode.

minimal on-axis longitudinal electric field, as well as simultaneous acceleration and deceleration near the waveguide edges. The side coupler design shown in Fig. 5(a) is found to be capable of exciting both of the two eigenmodes in Fig. 6, producing an electromagnetic field in the accelerating waveguide that is a composition of these two modes. Depending on the number of woodpile cells, the ratio of each mode in the composition changes. Cases of different numbers of cells have different mix ratio, thus an exact common point for traveling-wave launch could not occur.

Still, a point can be picked within the intersection area in Fig. 5(b), and a perturbed silicon guide launcher could be designed based on the corresponding $S_{22}{ }^{\mathrm{m}}$. With the two tuning dimensions introduced when describing the inset of Fig. 4(b), $l_{x}=82 \mathrm{~nm}$ and $z_{0}=863 \mathrm{~nm}$ give the desired $S_{22}{ }^{\mathrm{m}}$ of 0.849 and $323^{\circ}$ as its complex magnitude and phase, respectively. However, linking this silicon guide launcher back to back to the side couplers of a 7-cell accelerating waveguide structure results in significant reflection, as well as almost $50 \%$ normalized backward wave amplitude $|R|$ in the accelerating waveguide section. In Fig. 7(a), the effect of varying $l_{x}$ value (while $z_{0}$ stays at $863 \mathrm{~nm})$ on $\left|S_{21}{ }^{\text {tot }}\right|$ of the whole structure and $|R|$ in the accelerating waveguide section is shown. Both figures of merit exhibit a local extremum as $l_{\mathrm{x}}$ changes; however, these two extrema do not occur at the same $l_{\mathrm{x}}$ value. At $l_{\mathrm{x}}=66 \mathrm{~nm}$, the total power transmission is nearly $80 \%$, while the averaged $|R|$ in the accelerating waveguide is below 0.2. Curves in Fig. 7(b) plots the phase advance per cell $\psi$ (left ordinate) and $|R|$ (right ordinate) with respect to the longitudinal coordinate of the accelerating waveguide. They are not flat lines due to the mix of the fundamental and spurious propagating modes. At the simulated frequency $197 \mathrm{THz}$, the speed-of-light synchronous condition requires a phase advance per cell of $133.5^{\circ}$. The median $\psi$ value of the simulated structure is about $141^{\circ}$, which then needs to be further tuned down by decreasing the working frequency to achieve approximate phase synchronicity. In practice, tuning on the reflection magnitude (mainly determined by $l_{\mathrm{x}}$ ) and phase (mainly determined by $z_{0}$ ) of the side-coupling silicon guide could be achieved by
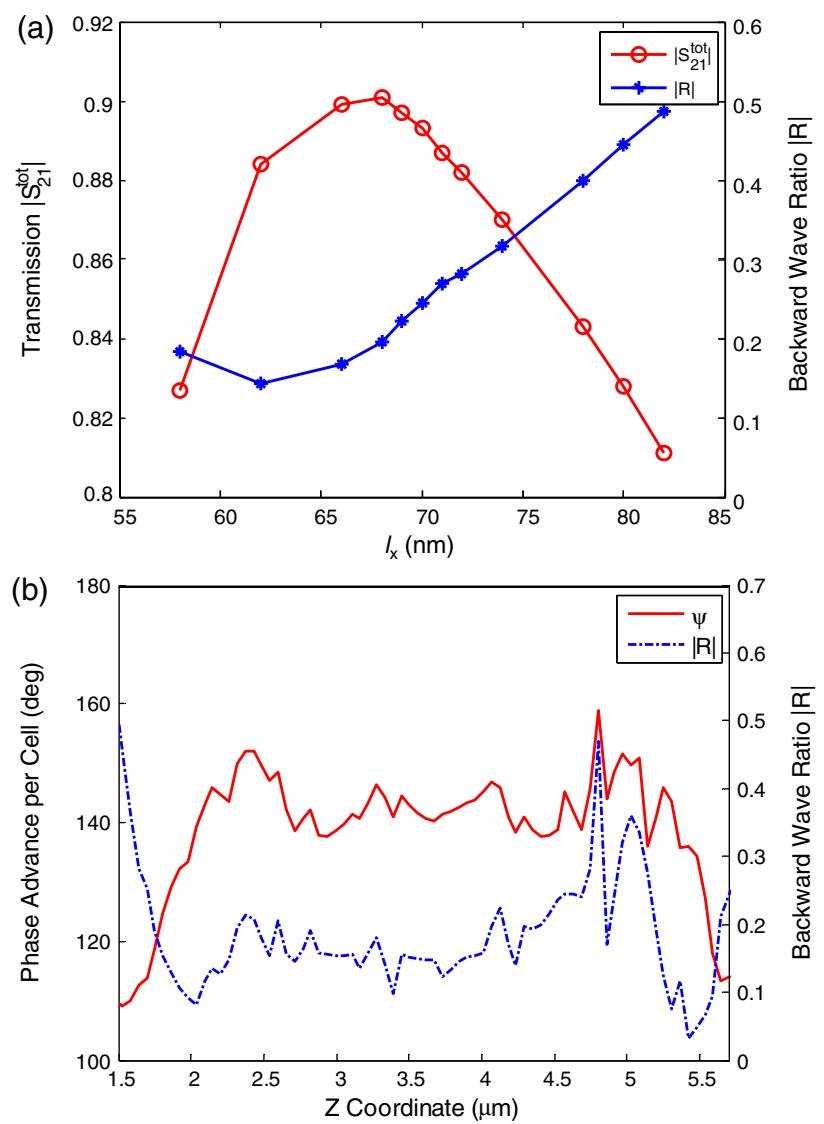

FIG. 7. (a) Effect on the total transmission $\left|S_{21}\right|$ and the normalized backward wave amplitude $|R|$ in the accelerating waveguide as the reflection magnitude $\left|S_{22} \mathrm{~m}\right|$ of the mode launcher varies. (b) The phase advance per cell and the normalized backward wave amplitude as the mode launcher uses the tuning dimensions $l_{\mathrm{x}}=66 \mathrm{~nm}$ and $z_{0}=863 \mathrm{~nm}$.

microheating elements [32,33], liquid crystal [34], or microelectromechanical systems devices [32,35] on silicon. Such tuning mechanisms will be important for achieving an efficient traveling wave match in a practical manufactured structure, due to fabrication errors and thermal drift. By scanning the working frequency and $l_{\mathrm{x}}$ and $z_{0}$ values, it is possible that a "best-scenario" coupling could be achieved spontaneously, with optimal power transmission and phase synchronicity, and minimal standing wave. However, a $100 \%$ traveling-wave launch of the desired fundamental accelerating mode is still preferable, not only as a good design reference, but also for its expandability to many cells, and for its suppression of the spurious mode, which wastes laser energy.

\section{B. A new accelerating mode and corresponding power coupler design}

An intuitive way to eliminate unwanted modes is to reduce the aperture size of the accelerating waveguide. With smaller channel width and height, and some modification of the silicon interface at the channel wall, several 
TABLE I. Characters of new TM-like propagating modes resulted from waveguide channel dimension variation. Their group velocity $V_{G}$ and phase velocity $V_{\Phi}$ are listed in units of the speed-of-light constant $c_{0}$.

\begin{tabular}{lccc}
\hline \hline $\begin{array}{l}\text { Mode } \\
\text { number }\end{array}$ & $\begin{array}{c}\text { Channel } \\
\text { aperture size }\end{array}$ & $V_{G} / c_{0}$ & $V_{\Phi} / c_{0}$ \\
\hline 1 & $2 a$ by $5 h$ & 0.3085 & 1.9692 at $217.83 \mathrm{THz}$ \\
2 & $2 a-w$ by $5 h$ & 0.2736 & 1.9403 at $214.63 \mathrm{THz}$ \\
3 & $2 a-0.825 a$ by $5 h$ & 0.0974 & 1.0014 at $192.01 \mathrm{THz}$ \\
\hline \hline
\end{tabular}

new TM-like confined modes are found, with their traits listed in Table 1. With a full channel aperture size of $2 a$ by $5 h$, TM mode 1 has its major electric field component along the longitudinal direction, as its vector field plot in Fig. 8(a) demonstrates. The electric field is also concentrated in the hollow core, following a Gaussian distribution that is ideal for electron acceleration. Its dispersion curve shown in Fig. 8(d) (black triangled line), however, is located far above the speed-of-light line (blue straight line), and would not intersect with it within the woodpile band-gap frequency range of roughly 180-227 THz (light-blue shaded area). Notice that the longitudinal phase velocity $k_{\mathrm{z}}$ in Fig. 8(d) is converted to phase advance per cell in degrees using the constant longitudinal length $a$ of one waveguide cell. This mode has a group velocity of about $31 \%$ of the speed of light, causing spatial "slippage" of the laser pulse energy from the electron bunch if employed for

(a) Mode 1

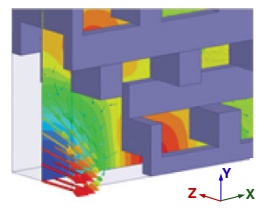

(b) Mode 2

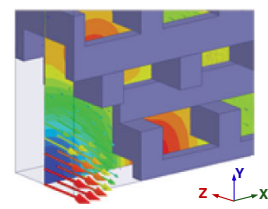

(d)

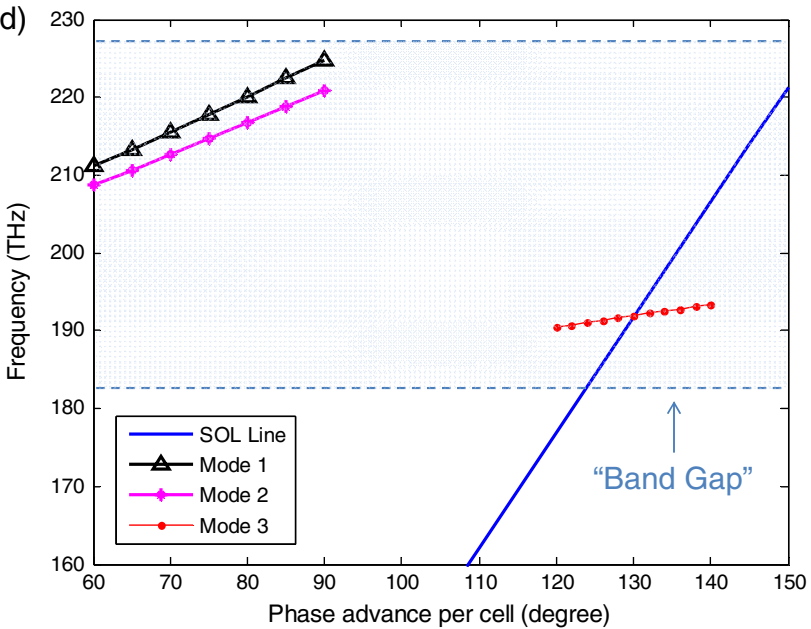

FIG. 8. New TM-like propagating modes of different woodpile waveguide channel dimensions, with their vector electric fields plotted in (a) mode 1, (b) mode 2, and (c) mode 3, and dispersion curves plotted in (d). acceleration. A full aperture size of $2 a-w$ by $5 h$ yields a similar TM mode 2 in Fig. 8(b), and a shifted-down dispersion curve [magenta line in Fig. 8(d)] in frequency. These two TM modes have favorable longitudinal electric fields, but the phase synchronicity requirement to a relativistic electron bunch rules them out as candidate accelerating modes, because they are too far from the SOL line to intersect within the band gap. The third design shown in Fig. 8(c) sweeps the vertical silicon wall of the second design towards the channel center by $233 \mathrm{~nm}$, giving a full aperture size of $2 a-0.825 a$ by $5 h$. This thickening of the silicon interface initiates a new TM-like mode, as the vector plot shows having a relatively uniform longitudinal electric field in the waveguide channel, and having its dispersion curve [red dotted line in Fig. 8(d)] crossing the SOL line at about $192 \mathrm{THz}$. Its group velocity, however, is further reduced to only about $10 \%$ of the speed of light, implying an even more severe laser pulse energy slippage than for modes 1 and 2. Also, its acceleration factor, defined as the averaged longitudinal gradient at the optimal injection phase, divided by the maximum electric field magnitude in silicon, is $69 \%$ of that of the original accelerating mode depicted in Fig. 6(a). All three TM-like modes are found to be the only modes excited in their respective waveguide channels by the side coupler layout introduced in Fig. 5(a). Since mode 3 is the most favorable for relativistic particle acceleration for the reasons just mentioned, we proceed now to simulate a traveling-wave launch of this mode near its phase synchronous frequency.

The back-to-back coupler layout shown in Fig. 9(a) is simulated to implement the same multiple-cell travelingwave launch method. The layout shown corresponds to an accelerating waveguide case with 7 cells, not counting the incomplete transitional cells at the ends where the input and output power coupling guides join with the accelerating guide. As before, the structure is excited by $z$-direction polarized TE modes of the side coupling silicon guides but without the quarter-wave impedance matching layers at the silicon guide ends that were seen in Fig. 5(a) (whose absence adds a reflection coefficient that can be incorporated to the mode launcher). As in Fig. 5(a), the model is a quarter-symmetric structure, with its front $Y Z$ plane and bottom $Z X$ plane assigned the perfect magnetic boundaries to simulate TM-like accelerating modes. The full width and the ending $x$ location of the coupling silicon guide is $w_{\text {coupler }}=a$ and $l_{\mathrm{Si}}=6.4 a$, respectively. The simulated electric field magnitude at the cross section near the middle of the accelerating waveguide is plotted (with two adjacent woodpile cells rendered transparent to give a better view), and it closely resembles the field distribution of the new TM-like mode supported by this accelerating channel design, as shown in Fig. 8(c), mode 3. In Fig. 9(b), the resultant $S_{22}{ }^{\mathrm{m}}$ magnitude vs phase curves of structures with 6,7 , and 8 cells of accelerating waveguides are plotted. They do not exactly intersect at one point. However, 

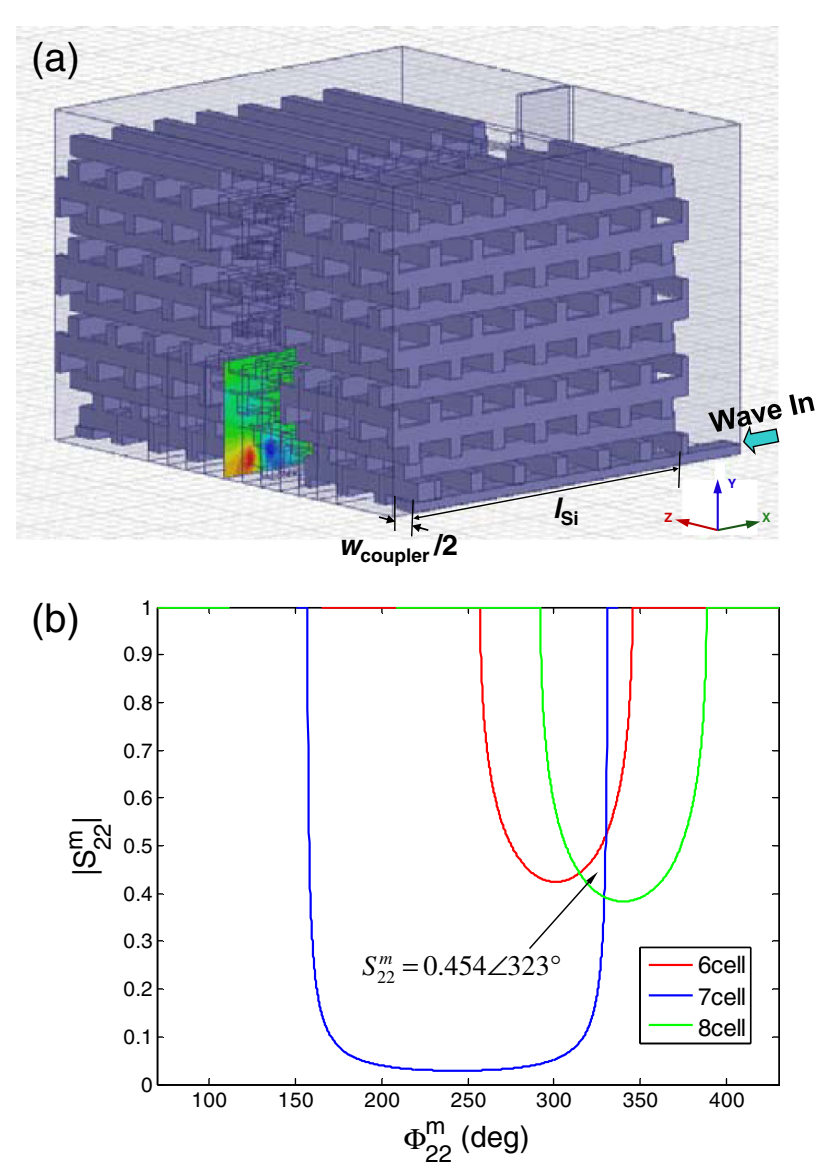

FIG. 9. (a) TE-to-TM mode power coupler design for the new accelerating mode, and (b) its multiple-cell simulation results to determine the required $S_{22}$ m for traveling-wave match.

observing this intersecting area is small enough and with the confidence from the single-mode situation in the accelerating waveguide, an $S_{22}$ m value in the middle of the intersecting area is still chosen [as marked in Fig. 9(b)] to design the traveling-wave mode launcher upon.

Using the same perturbed silicon guide design as the inset of Fig. 4(b) illustrates, dimensions of the embedded PEC chunk are determined to be $l_{\mathrm{x}}=45 \mathrm{~nm}$ and $z_{0}=826 \mathrm{~nm}$ to yield an $S_{22}{ }^{\mathrm{m}}$ of 0.454 magnitude and $323^{\circ}$ phase. Adding this mode launcher back to back to the 8-cell power coupler structure, the simulated total power reflection at $191.35 \mathrm{THz}$ is less than $-35 \mathrm{~dB}$, and the total power transmission is $-0.13 \mathrm{~dB}$. The excited electric field in the accelerating waveguide again replicates the new propagating SOL mode from eigenmode simulation. Calculation results of $\psi$ and $|R|$ using the extracted on-axis longitudinal electric field are plotted in Fig. 10. They are both relatively flat in the middle waveguide region, with about a $125^{\circ}$ phase advance per cell very close to phase synchronous condition, and about $4 \%$ normalized backward wave amplitude. For a 6-cell structure, simulation gives similar results of $-0.19 \mathrm{~dB}$ total power transmission to the fundamental accelerating mode, and less than 5\% normalized backward wave amplitude. Therefore, a

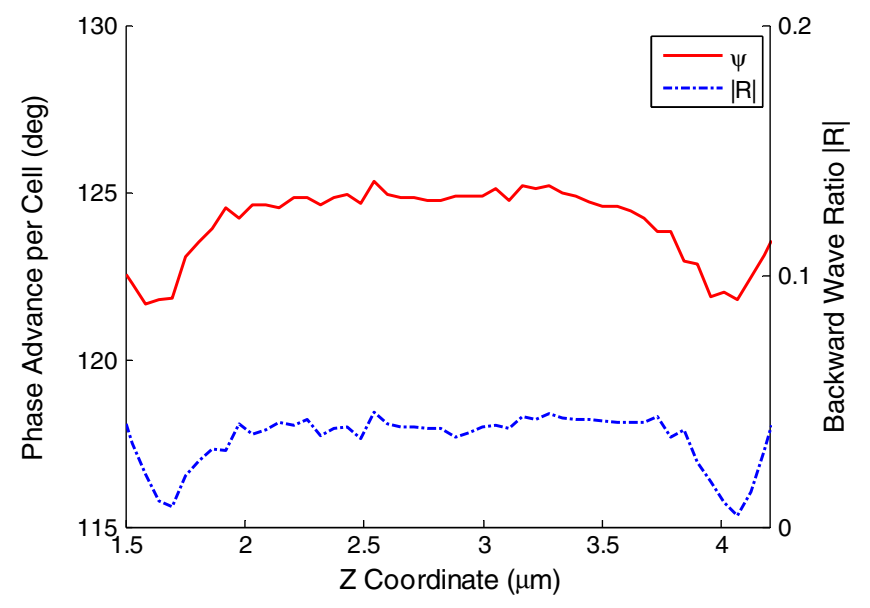

FIG. 10. Phase advance per cell $\psi$ and normalized backward wave amplitude $|R|$ of an 8-cell power coupler structure, proving it is traveling-wave matched to the new woodpile accelerating mode.

traveling wave of the new accelerating mode is indeed launched in the woodpile waveguide, with $95 \%-96 \%$ of the full input power coupled to it.

\section{DISCUSSION AND CONCLUSION}

In this work we use frequency domain finite element electromagnetic simulations to show that silicon-guidebased side couplers can couple power efficiently into a three-dimensional photonic crystal waveguide for acceleration of relativistic charged particles. Two methods developed originally for design of conventional metallic rf-cavity accelerators are adapted to this purpose, and detailed calculations are conducted for the example of a threedimensional photonic crystal based upon the silicon woodpile design. A traveling-wave excitation of the desired accelerating mode with a negligible standing-wave ratio is successfully achieved. These calculations show the feasibility of TE-to-TM-mode coupling between the silicon power distribution network and the woodpile accelerating waveguide, which is a key component for an on-chip three-dimensional woodpile laser accelerator. This approach can also potentially be employed for coupling to three-dimensional photonic crystal waveguides based upon other geometries, made from other materials, or designed for other types of traveling wave modes.

Single-mode operation in the accelerating waveguide is found to be critical for the traveling-wave launch method to work properly. The original accelerating waveguide design allows a spurious higher-order TM mode to propagate. Therefore a pure traveling-wave launch from side couplers is not successful. Reducing the channel aperture size and engineering the silicon/air interface of the accelerating waveguide reveals a new accelerating mode upon which traveling-wave propagation is established, with up to 96\% power coupling efficiency into this mode from side 
couplers. This new accelerating mode has its acceleration factor just $69 \%$ of that of the original accelerating mode design. Also from its dispersion curve shown in Fig. 8(d), this mode has a group velocity only about one-tenth of the speed of light, implying faster energy "slippage" of the laser wave packet from the electron bunch. Therefore more frequent couplers along the accelerating waveguide are needed to gain the same particle energy. Reduced waveguide channel size means less unscattered electron throughput as well. For these reasons, preferential excitation of the fundamental accelerating mode in an overmoded woodpile waveguide is worth exploration, possibly by special designs at the coupling region or multiple coupler feeds. Full structure simulation instead of utilizing the four-fold symmetry needs to be carried out as well.

Damage threshold of the building materials of these accelerator structures under pulsed laser determine how hard they can be driven by the incoming laser field. Nonlinear effects occur when high density photons are directly shed upon the material, such as multiphoton ionization, thermal ablation, and inverse bremsstrahlung [36-38]. Silicon is not an ideal material to start with in this sense, due to its semiconductor nature and relatively low band-gap frequency $(\sim 1100 \mathrm{~nm})$. Studies of pulsed laser breakdown of various dielectrics conducted in our group [39] reveal a damage threshold fluence of merely $0.19 \mathrm{~J} / \mathrm{cm}^{2}$ for silicon, under illumination of about 1 picosecond long, $800 \mathrm{~nm}$ wavelength, $600 \mathrm{~Hz}$ laser pulses. This number is $3.5 \mathrm{~J} / \mathrm{cm}^{2}$ for fused silica and $4.9 \mathrm{~J} / \mathrm{cm}^{2}$ for sapphire under the same excitation condition. In general, higher band-gap materials such as $\mathrm{SiO}_{2}(\sim 140 \mathrm{~nm})$, $\mathrm{Al}_{2} \mathrm{O}_{3}(\sim 140 \mathrm{~nm})$, and $\mathrm{CaF}_{2}(\sim 103 \mathrm{~nm})$ exhibit better damage thresholds, because they stay further away from photon absorption of the drive laser. However, silica only possess a refractive index of about 1.44, and thus not enough contrast with respect to air to build a complete three-dimensional band-gap photonic crystal waveguide to regulate the photons [14]. On the other hand, sapphire has a large enough refractive index of about 3 but is a challenging material to handle in lithographic processes. Considering the refractive index contrast and manufacturing readiness, silicon is still an intriguing material to build threedimensional photonic crystal laser accelerators upon, especially with the advancement of laser energy and stability towards mid-infrared wavelengths where it can withstand higher fluence.

At $1.55 \mu \mathrm{m}$ excitation wavelength, data from our previous study [39] shows about $0.35 \mathrm{~J} / \mathrm{cm}^{2}$ damage threshold of silicon, about a factor of 2 improvement over $800 \mathrm{~nm}$. The side coupler design presented in this work, relying on an inserted silicon guide to deliver laser energy directly to the air core of the woodpile accelerating waveguide, creates localized "hot spots" in silicon near the coupling corner that are more vulnerable places to laserinduced damage. Our latest optimized coupler design yields an acceleration factor of roughly 0.13 of a coupled full woodpile accelerator structure, including the couplers and the accelerating waveguide. Given 1 picosecond pulse duration and this acceleration factor, it translates to $134 \mathrm{MV} / \mathrm{m}$ maximum acceleration gradient. Possibilities to enhance this maximum acceleration gradient include building just the coupler guide section with materials of much higher damage thresholds (e.g., silica and sapphire), using a shorter laser pulse, or driving the accelerator structure at a longer wavelength thus further away from the silicon band gap.

As long as manufacturing of the structure is concerned, the coupler region within the woodpile structure could be straightforwardly fabricated by photolithography since it does not involve material other than silicon. A new approach to form three-dimensional structures by transferring and stacking prepatterned, freestanding silicon membranes is being experimented, and details of this approach can be found in the Appendix. In the current design, perturbation required in the silicon-guide mode launcher is realized by obstacles of perfect conductors, which are undesirable in an actual physical structure. An air discontinuity in the silicon guide with carefully tuned dimensions $[19,28]$ might just as well fulfill that task. The more challenging part left is then the transition between the on-substrate silicon power network and the silicon side-coupling guide next to the woodpile structure. A free-space coupling of light into these side couplers is possible for prototyping characterization experiments, although sub- $\mu \mathrm{m}$ scale alignment precision is required. Integrated fabrication of the whole structure is favorable and under pursuit.

\section{ACKNOWLEDGMENTS}

This work is supported by the U.S. Department of Energy under Grants No. DE-AC02-76SF00515 and No. DE-FG06-97ER41276 and by DARPA Grant No. N66001-11-1-4199.

\section{APPENDIX: WOODPILE ACCELERATING WAVEGUIDE FABRICATION}

Previous work has been successful in fabricating a silicon woodpile accelerating waveguide, using layer-bylayer photolithographic process [22] or a combination of direct laser writing and silicon double inversion [40]. While the layer-by-layer lithographic method provides ideal rectangular definition of the silicon rods and the rectangular channel edges, and is well suited to rapid mass production once a suitable fabrication process is established, the stacking of many layers ( $>10)$ proves challenging for purposes of prototyping test devices for demonstration experiments. The double-inversion process is not limited on the number of layers; however, the silicon rods are opal shaped, and the channel edge is rounded due to the profile 


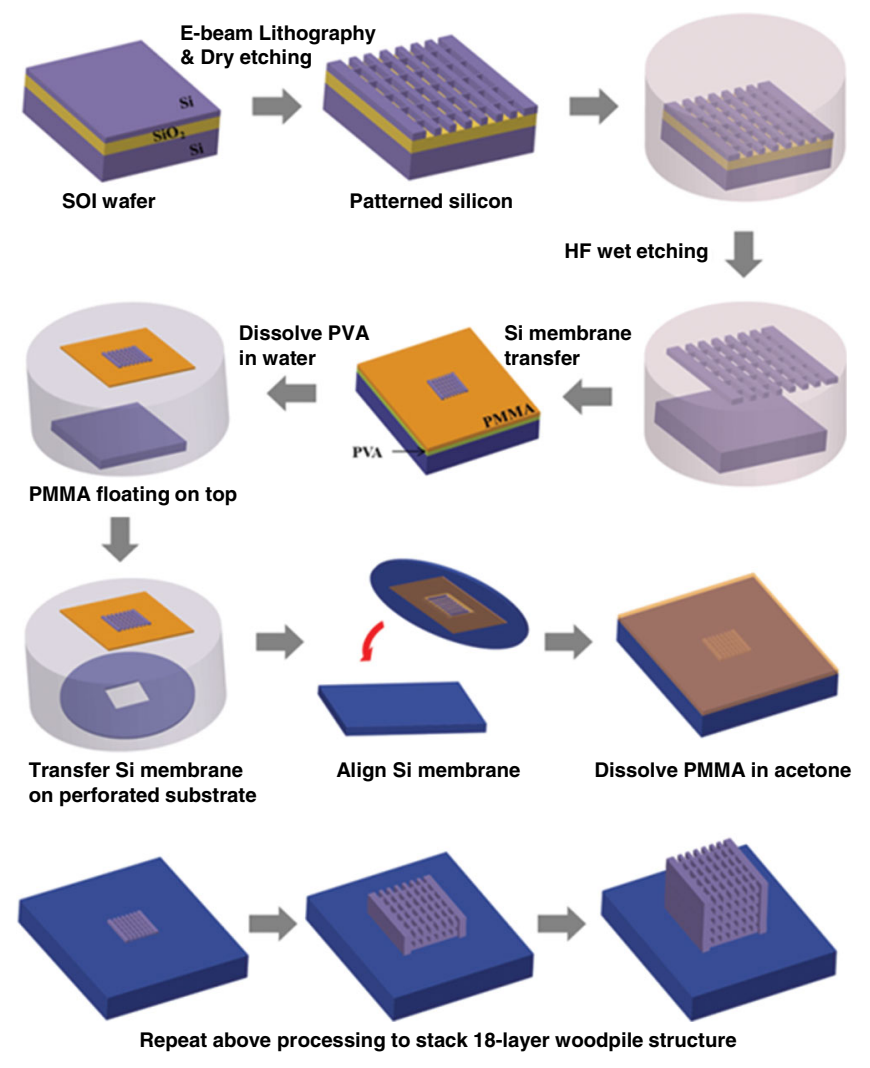

FIG. 11. Schematic representation of the silicon membrane transfer method to stack multiple woodpile layers.

of the focused ion beam used for milling it open. Currently, a new approach to form three-dimensional structures by transferring and stacking prepatterned, freestanding silicon membranes is being implemented to fabricate woodpile accelerating structures. This technique does not require high temperature processes, allows flexibility in the choice of materials, and allows for prototyping of woodpile stacks with 10-20 layers.

The overall fabrication process is depicted in Fig. 11. The woodpile pattern is first etched on the top layer of an SOI wafer, and then released from the substrate with hydrofluoric acid wet etch. The released membrane is then transferred on top of a polymethyl methacrylate (PMMA) film, separated from a silicon substrate by a polyvinyl alcohol (PVA) film. Dipping the silicon wafer in water dissolves PVA and floats the PMMA film with woodpile membrane attached. Then the PMMA/woodpile film is again mounted on a handling silicon wafer with an opening at the center where the woodpile structure will be located. This handling wafer will be flipped with the PMMA/woodpile facing down and then aligned and stacked onto a substrate with previously transferred woodpile layers. After dissolving the PMMA layer in acetone, more woodpile layers are stacked on.

We recently used this approach to successfully fabricate an 18-layer silicon woodpile photonic crystal, and other cavity and waveguide structures. Figure 12(a) shows a (a)

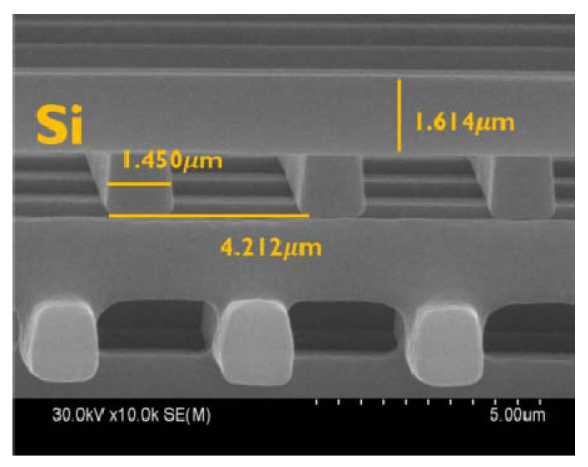

(b)

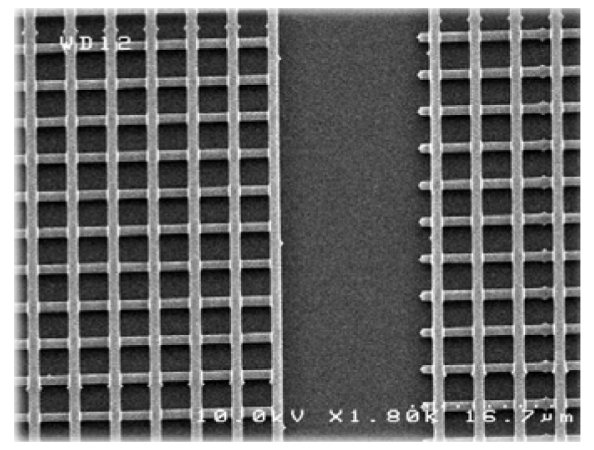

FIG. 12. (a) Side SEM view of an 18-layer silicon woodpile structure. (b) Top SEM view of the middle layers of a woodpile waveguide structure. Both are fabricated by the silicon membrane method.

cross-sectional scanning electron micrograph (SEM) image of an 18-layer stacked silicon woodpile in the mid-infrared frequency range, with $a, w$, and $h$ dimensions of $4.2 \mu \mathrm{m}$, $1.4 \mu \mathrm{m}$, and $1.6 \mu \mathrm{m}$, respectively. Figure 12(b) shows a top SEM view of the middle two layers of a near-infrared woodpile waveguide structure that we are presently fabricating at Stanford, leaving the central channel as the accelerating waveguide. Attempts are being made to fabricate widened silicon rod side couplers that extend out of the woodpile structure, similar to the layout shown in Fig. 9(a). The extended part could potentially be held by low-index-material (e.g. silicon dioxide) webbing links, grown from adjacent woodpile rods to help support it.

The current woodpile structure in fabrication has a target wavelength scaled up from the nominal $1.55 \mu \mathrm{m}$ to $3.4 \mu \mathrm{m}$, corresponding to dimensions of $a=1.2 \mu \mathrm{m}, w=344 \mathrm{~nm}$, and $h=440 \mathrm{~nm}$. The target waveguide channel will be $2 a-w$ wide, $5 h$ tall, and $101 \mu \mathrm{m}$ long (84 periods). Eight layers of silicon rods on each side of the waveguide channel will be fabricated to provide sufficient cladding, making a 21-layer stacked structure. The manufactured sample will be characterized by SEM imaging, and infrared spectroscopy to verify its photonic band-gap frequency and bandwidth. The waveguide mode will be characterized first by laser coupling transmission measurements (for TE modes), and then by spectroscopic measurement of wakefield radiation induced by an electron beam traversing the accelerating channel (for TM modes). Future fabrication 
efforts will address integration of the coupling waveguides, and scale towards a shorter operating wavelength in the range of $1.5-2 \mu \mathrm{m}$, which would provide greater compatibility with available infrared solid state laser sources.

[1] T. Plettner P. P. Lu, and R. L. Byer, Phys. Rev. ST Accel. Beams 9, 111301 (2006).

[2] X. Lin, Phys. Rev. ST Accel. Beams 4, 051301 (2001).

[3] K. Shimoda, Appl. Opt. 1, 33 (1962).

[4] M. A. Piestrup, G. B. Rothbart, R. N. Fleming, and R. H. Pantell, J. Appl. Phys. 46, 132 (1975).

[5] R. B. Palmer, J. Appl. Phys. 43, 3014 (1972).

[6] A. Van Steenbergen, J. Gallardo, J. Sandweiss, and J.-M. Fang, Phys. Rev. Lett. 77, 2690 (1996).

[7] Y. Takeda and I. Matsui, Nucl. Instrum. Methods 62, 306 (1968).

[8] K. Mizuno, J. Pae, T. Nozokido, and K. Furuya, Nature (London) 328, 45 (1987).

[9] J. Breuer and P. Hommelhoff, Phys. Rev. Lett. 111, 134803 (2013).

[10] E. A. Peralta, E. Colby, R. J. England, C. McGuinness, B. Montazeri, K. Soong, Z. Wu, and R. L. Byer, AIP Conf. Proc. 1507, 169 (2012).

[11] A. Mizrahi and L. Schachter, Phys. Rev. E 70, 016505 (2004).

[12] B. M. Cowan, Phys. Rev. ST Accel. Beams 11, 011301 (2008).

[13] E. A. Peralta, K. Soong, R. J. England, E. R. Colby, Z. Wu, B. Montazeri, C. McGuiness, J. McNeur, K. J. Leedle, D. Walz, E. B. Sozer, B. Cowan, B. Schwartz, G. Travish, and R. L. Byer, Nature (London) 503, 91 (2013).

[14] J. D. Joannopoulos, S. G. Johnson, J. N. Winn, and R. D. Meade, Photonic Crystals: Molding the Flow of Light, 2nd ed. (Princeton University Press, Princeton, NJ, 2008).

[15] K. Soong, E. R. Colby, C. McGuinness, R. L. Byer, and E. A. Peralta, in Proceedings of the 24th Particle Accelerator Conference, PAC-2011, New York (IEEE, New York, 2011), p. 277, C110328.

[16] C. McGuinness, E. Colby, and R. L. Byer, J. Mod. Opt. 56, 2142 (2009).

[17] B. Cowan, M. C. Lin, B. T. Schwartz, E. R. Colby, R. J. England, R. J. Noble, J. E. Spencer, R. L. Byer, and C. M. McGuinness, in Proceedings of the International Particle Accelerator Conference, Kyoto, Japan, 2010 (IEEE, Kyoto, Japan, 2010), C100523, THPEC013.

[18] B. Cowan, Ph.D. thesis, Stanford University, 2007.
[19] Z. Wu, C. Ng, C. McGuinness, and E. Colby, in Proceedings of the 24th Particle Accelerator Conference, PAC-2011, New York (IEEE, New York, 2011), MOP072.

[20] K. Soong and R. L. Byer, Opt. Lett. 37, 975 (2012).

[21] Z. Wu, "Dipole modes in a silicon woodpile structure for sub-micron beam position monitoring" (unpublished).

[22] R. H. Siemann, Phys. Rev. ST Accel. Beams 7, 061303 (2004).

[23] C. McGuinness, Ph.D. thesis, Stanford University, 2012.

[24] B. M. Cowan, Design and Fabrication of ThreeDimensional Photonic Crystal Accelerator Structures, FY 2010 SBIR/STTR Phase II, Topic 38.a, Tech-X Corporation, Boulder, CO, 2010.

[25] T. Plettner and R. L. Byer, Phys. Rev. ST Accel. Beams 11, 030704 (2008).

[26] Z. Wu, R. J. England, C. Ng, and S. G. Tantawi, AIP Conf. Proc. 1507, 535 (2012).

[27] D. Vermeulen S. Selvaraja, P. Verheyen, G. Lepage, W. Bogaerts, P. Absil, D. Van Thourhout, and G. Roelkens, Opt. Express 18, 18278 (2010).

[28] H. T. Chen and K. J. Webb, Opt. Lett. 31, 2145 (2006).

[29] N. M. Kroll, C.-K. Ng, and D. C. Vier, Report No. SLACPUB-8614, 2000.

[30] High Frequency Structure Simulator, version 13, ANSYS Corporation.

[31] C. Nantista, S. Tantawi, and V. Dolgashev, Phys. Rev. ST Accel. Beams 7, 072001 (2004).

[32] R. Soref, IEEE J. Sel. Top. Quantum Electron. 12, 1678 (2006).

[33] M. Pruessner, T. Stievater, M. Ferraro, and W. Rabinovich, Opt. Express 15, 7557 (2007).

[34] J. Cos, J. Ferré-Borrull, J. Pallarès, and L. F. Marsal, Opt. Quantum Electron. 42, 487 (2011).

[35] A. Lipson, Ph.D. thesis, University of London and the Diploma of Imperial College London, 2006.

[36] M. Lenzner, J. Kruger, S. Sartania, Z. Cheng, C. Spielmann, G. Mourou, W. Kautek, and F. Krausz, Phys. Rev. Lett. 80, 4076 (1998).

[37] A. Kaiser, B. Rethfeld, M. Vicanek, and G. Simon, Phys. Rev. B 61, 11437 (2000).

[38] Z. Duanming, L. Dan, L. Zhihua, H. Sipu, Y. Boming, G. Li, T. Xinyu, and L. Li, Physica B (Amsterdam) 362, 82 (2005).

[39] K. Soong, R. L. Byer, C. McGuinness, E. Peralta, and E. Colby, in Proceedings of the 24th Particle Accelerator Conference, PAC-2011, New York (IEEE, New York, 2011), MOP095.

[40] I. Staude, C. McGuinness, A. Frolich, R. L. Byer, E. Colby, and M. Wegener, Opt. Express 20, 5607 (2012). 